\title{
Modeling of Proton Exchange Membrane Fuel Cell Using Support Vector Regression Machine
}

\author{
Jiangling TANG, Jian HUANG* \\ Department of Physics and Engineering, Guilin normal college, Guilin, Guangxi 541001, P.R. China \\ *email: Tantian62@hotmail
}

Keywords: Support Vector Regression Machines; Voltage; Proton Exchange Membrane Fuel Cell; Modeling

\begin{abstract}
In this paper, a nonlinear offline model of proton exchange membrane fuel cell (PEMFC) is built by using a support vector regression machine (SVRM) based on particle swarm optimization (PSO) algorithm. During the process of modeling, the PSO aims to optimize the parameters of SVRM. Compared with the artificial neural network (ANN) approach, the prediction results show that the SVRM approach is superior to the conventional ANN in predicting the stack voltage with different hydrogen pressure. The mean absolute percentage error (MAPE) of 36 test samples is $0.73 \%$, such that prediction result was provided by leave-one-out cross validation (LOOCV) test of SVRM. So it is feasible to establish the prediction model of PEMFC system by using SVRM identification based on the PSO.
\end{abstract}

\section{Introduction}

Fuel cell, as an electrochemical device, can directly convert the chemical energy to electrical energy and thermal energy without burning. Among the various available fuel cells, the proton exchange membrane fuel cell (PEMFC) is the focus of current development efforts because it is capable of low operating temperature, low emission, high power density, high efficiency, fast startup and quick response. The PEMFC system is a nonlinear, multi-variable system , a sound model can help size, simulate, evaluate and optimize PEMFC research programs [1], and is thus of great importance to users and researchers.

In the last several decades, fruitful results from PEMFC system modeling have been obtained. But, most of the existing models focus on the design of the PEMFC [2] [3]. What matters most to PEMFC users, however, are not PEMFC relevant internal details but its performance under different operating conditions. What users really need are prediction models, with which they can predict the PEMFC performance under various operating conditions. To meet the demand of this, some researchers have attempted to establish novel PCMFC models. Black-box identification technique such as the artificial neural network (ANN) has been used to derive a PEMFC model from the experimental data [4] [5]. The artificial neural network has the ability to learn and approach the nonlinear function, and has been considered as a powerful computing tool for establishing the mathematical relationship of the nonlinear system based on the input-output data. But, ANN has the following insurmountable shortcomings: lack of a unified mathematical theory; easy to enmesh local minimization; weakly generalization ability for the small-sample dataset; prone to over-fitting, etc.

Support vector regression machine (SVRM), proposed by Vapnik and co-workers, is a new powerful statistical learning approach base on structural risk minimization (SRM) and Vapnik-Chevronenks dimensions principle [6] [7]. Research shows that predictive results of SVRM with many excellences, such as fast-learning, global optimization and excellent generalization ability for the small-sample dataset, are generally superior to those of other pattern recognitions and regressions. At present, SVRM has become a focus in machine learning research and is employed in a wide range of prediction problems [8] [9] [10]. In this paper, the SVRM prediction model, based on particle swarm optimization (PSO) algorithm for its parameter optimization, and integrating leave-one-out cross validation (LOOCV), was set up according to the PEMFC stack voltage dataset, 
which was measured under different $\mathrm{H}_{2}$ pressure by N. Bhoopal et al. [11], and its predictive errors were compared with that of ANN model.

\section{Description of the PEMFC}

The typical structure of a single PEMFC is consists of anode, electrolyte membrane and cathode.The electrochemical reactions at the anode and cathode in a single PEMFC is below:

Anode reactions: $\quad \mathrm{H}_{2} \rightarrow 2 \mathrm{H}^{+}+2 \mathrm{e}^{-}$

Cathode reactions: $\quad \mathrm{O}_{2}+4 \mathrm{H}^{+}+4 \mathrm{e}^{-} \rightarrow 2 \mathrm{H}_{2} \mathrm{O}$

Overall reactions: $\quad 2 \mathrm{H}_{2}+\mathrm{O}_{2} \rightarrow 2 \mathrm{H}_{2} \mathrm{O}$

On the anode side, hydrogen molecule splits up into two hydrogen protons and two electrons. Electrons trough an external circuit and form current, while protons migrate through the electrolyte membrane. On the cathode side, the oxygen reacts with protons coming from anode and electron coming from external circuit, and finally produces with water and heat.

\section{Theory of SVRM}

In SVRM, the basic idea is to map $\boldsymbol{X}$ from the input space into a higher-dimensional feature space $\boldsymbol{F}$ via a nonlinear mapping function $\Phi(\boldsymbol{x})$, and then to conduct linear regression in $\boldsymbol{F}$ space. Therefore, SVRM is to find the linear relation Eq. (1) based on a given dataset $\left(\boldsymbol{x}_{1}, y_{1}\right), \ldots,\left(\boldsymbol{x}_{n}, y_{n}\right)$.

$$
f(\boldsymbol{x})=\boldsymbol{w} \cdot \Phi(\boldsymbol{x})+b, \Phi: \boldsymbol{R}^{n} \rightarrow \boldsymbol{F}, \boldsymbol{w} \in \boldsymbol{F} .
$$

Where $\boldsymbol{w}$ is a vector for regression coefficients in $\boldsymbol{F}, b$ is a bias. They are estimated by minimizing the regularized risk function $R(C)$, namely:

$$
\begin{aligned}
& \text { minimize } R(C)=\frac{1}{2}\|\boldsymbol{w}\|^{2}+C \sum_{i=1}^{n} L_{\varepsilon}\left(f\left(\boldsymbol{x}_{i}\right)-y_{i}\right), \\
& L_{\varepsilon}\left(f\left(\boldsymbol{x}_{i}\right)-y_{i}\right)=\left\{\begin{array}{l}
0, \quad \text { if }\left|f\left(\boldsymbol{x}_{i}\right)-y_{i}\right|<\varepsilon, \\
\left|f\left(\boldsymbol{x}_{i}\right)-y_{i}\right|-\varepsilon, \quad \text { if }\left|f\left(\boldsymbol{x}_{i}\right)-y_{i}\right| \geq \varepsilon .
\end{array}\right.
\end{aligned}
$$

Where $n$ is the number of training samples, $\varepsilon$ is the maximum deviation allow during the training and $C$ represents the associated penalty for excess deviation during the training, $(1 / 2)\|\boldsymbol{w}\|^{2}$ is used as a measurement of function flatness. The second term in Eq. (2), $c \sum_{i=1}^{n} L_{\varepsilon}\left(f\left(x_{i}\right)-y_{i}\right)$ is the so called empirical risk and measured by $\varepsilon$-insensitive loss function $L_{\varepsilon}\left(f\left(\boldsymbol{x}_{i}\right)-y_{i}\right)$, which indicates that it does not penalize errors below $\varepsilon$.

By minimizing Eq.(2), the array $\boldsymbol{w}$ can be as:

$$
\boldsymbol{w}=\sum_{i=1}^{l}\left(\alpha_{i}-\alpha_{i}^{*}\right) \Phi\left(\boldsymbol{x}_{i}\right) .
$$

where $l$ is the number of SVs. Finally, the linear relation Eq. (1) has the following explicit form:

$$
f(x)=\sum_{i=1}^{l}\left(\alpha_{i}-\alpha_{i}^{*}\right) k\left(x, x_{i}\right)+b,
$$

In Eq. (5), $k\left(x, x_{i}\right)=\Phi(x) \cdot \Phi\left(x_{i}\right)$ is a kernel function. Choosing different kernel function can generate different SVRM models. There are four commonly used kernel functions, i.e., linear kernel, radial basis kernel, polynomial kernel, sigmoid kernel, etc. In this paper, the radial basis kernel (6) was utilized:

$$
k\left(\boldsymbol{x}, \boldsymbol{x}_{i}\right)=\exp \left(-\gamma\left\|\boldsymbol{x}-\boldsymbol{x}_{i}\right\|^{2}\right)
$$




\section{The SVRM prediction model}

The datasets used in this study was generated by $\mathrm{N}$. Bhoopal et al., includes 36 samples. The detailed experimental procedure can be referred from Ref. [11].

For a given PEMFC stack, the relation between stack voltage $V$ and current density $I$ is influenced by many operating parameters, such as cell temperature, air flow rate, air pressure, hydrogen flow rate, hydrogen pressure, etc. However, due to the high number of operating variables, a complete experimental database of PEMFC under the different operating conditions is difficult to obtain and no data are available in the open literature yet [12] [13]. Up to now, almost no model has ever been able to accommodate all these operating variables. SVRM model is no exception. Hydrogen pressure is one of the most operating parameters for the fuel cell and has a significant effect on the fuel cell. In order to analyze the effects of different hydrogen pressure on output voltage, in our SVRM model, current density I and hydrogen pressure P were employed as input variables, while as stack voltage $V$ as output variables. The modeling and prediction were conducted by using the 36 samples based on SVRM approach via leave-one-out cross validation (LOOCV). Cross validation, sometimes called rotation estimation, is a technique for assessing how the results of a statistical analysis will generalize to an independent data set. It is mainly used in settings where the goal is prediction, and one wants to estimate how accurately a predictive model will perform in practice. As the name suggests, leave-one-out cross validation (LOOCV) involves using a single observation from the original sample as the validation data, and the remaining observations as the training data. This is repeated such that each observation in the sample is used once as the validation data.

\section{Results and analysis}

The indexes from the $5^{\text {th }}$ and $7^{\text {th }}$ columns in Table 1 illustrate the PEMFC stack voltage predicted by ANN prediction model and SVRM prediction model, respectively. Table 2 lists MAE, MAPE and $R^{2}$ of the prediction results by ANN and SVRM.

From Table 1, it can be observed that, among all 36 samples, there are 27 samples of SVRM prediction model, the absolute percentage errors are within a very small range of $0-0.50 \%, 6$ samples equal to zero and 15 samples only reach $0.01 \%$. The maximum absolute percentage error is not exceeded 5.00\%. This reveals that the precise of the SVRM models, which is small enough to meet the demand of experiments. 
Table 1: Measured and predicted results

\begin{tabular}{|c|c|c|c|c|c|c|c|}
\hline \multirow[b]{2}{*}{ NO } & \multirow[b]{2}{*}{$\begin{array}{c}\text { Current } \\
\text { (A) }\end{array}$} & \multirow{2}{*}{$\begin{array}{c}\mathrm{H}_{2} \\
\text { pressure } \\
\text { (Bar) }\end{array}$} & \multirow{2}{*}{$\begin{array}{c}\text { Practical } \\
\text { value } \\
\text { (V) }\end{array}$} & \multicolumn{2}{|c|}{ ANN } & \multicolumn{2}{|c|}{ SVRM } \\
\hline & & & & $\begin{array}{c}\text { Prediction } \\
\text { (V) }\end{array}$ & Error & $\begin{array}{l}\text { Prediction } \\
\text { (V) }\end{array}$ & Error \\
\hline 1 & 1.0000 & 0.2000 & 2.7000 & 2.6000 & $3.70 \%$ & 2.5981 & $3.77 \%$ \\
\hline 2 & 2.0000 & 0.2000 & 2.6000 & 2.5700 & $1.15 \%$ & 2.6000 & $0.00 \%$ \\
\hline 3 & 3.0000 & 0.2000 & 2.5000 & 2.5680 & $2.72 \%$ & 2.4999 & $0.00 \%$ \\
\hline 4 & 4.0000 & 0.2000 & 2.4000 & 2.4500 & $2.08 \%$ & 2.4001 & $0.00 \%$ \\
\hline 5 & 5.0000 & 0.2000 & 2.3000 & 2.2900 & $0.43 \%$ & 2.4000 & $4.35 \%$ \\
\hline 6 & 5.7000 & 0.2000 & 2.2000 & 2.1900 & $0.45 \%$ & 2.2001 & $0.01 \%$ \\
\hline 7 & 6.6000 & 0.2000 & 2.1000 & 2.0000 & $4.76 \%$ & 2.0999 & $0.00 \%$ \\
\hline 8 & 7.5000 & 0.2000 & 2.0000 & 2.0000 & $0.00 \%$ & 1.9998 & $0.01 \%$ \\
\hline 9 & 7.7000 & 0.2000 & 2.0000 & 2.0000 & $0.00 \%$ & 2.0001 & $0.00 \%$ \\
\hline 10 & 1.0000 & 0.4000 & 2.8000 & 2.9000 & $3.57 \%$ & 2.8002 & $0.01 \%$ \\
\hline 11 & 2.0000 & 0.4000 & 2.7000 & 2.7000 & $0.00 \%$ & 2.6955 & $0.17 \%$ \\
\hline 12 & 3.0000 & 0.4000 & 2.7000 & 2.6000 & $3.70 \%$ & 2.6605 & $1.46 \%$ \\
\hline 13 & 4.0000 & 0.4000 & 2.6000 & 2.5000 & $3.85 \%$ & 2.5998 & $0.01 \%$ \\
\hline 14 & 5.0000 & 0.4000 & 2.5000 & 2.4000 & $4.00 \%$ & 2.3989 & $4.05 \%$ \\
\hline 15 & 5.7000 & 0.4000 & 2.2000 & 2.2300 & $1.36 \%$ & 2.2521 & $2.37 \%$ \\
\hline 16 & 6.6000 & 0.4000 & 2.1000 & 2.1000 & $0.00 \%$ & 2.0998 & $0.01 \%$ \\
\hline 17 & 7.5000 & 0.4000 & 2.0000 & 2.0000 & $0.00 \%$ & 1.9997 & $0.01 \%$ \\
\hline 18 & 7.7000 & 0.4000 & 2.0000 & 2.0000 & $0.00 \%$ & 2.0010 & $0.05 \%$ \\
\hline 19 & 1.0000 & 0.5000 & 2.9000 & 2.8000 & $3.45 \%$ & 2.8998 & $0.01 \%$ \\
\hline 20 & 2.0000 & 0.5000 & 2.7000 & 2.6700 & $1.11 \%$ & 2.7003 & $0.01 \%$ \\
\hline 21 & 3.0000 & 0.5000 & 2.6000 & 2.4500 & $5.77 \%$ & 2.6002 & $0.01 \%$ \\
\hline 22 & 4.0000 & 0.5000 & 2.5000 & 2.5100 & $0.40 \%$ & 2.5003 & $0.01 \%$ \\
\hline 23 & 5.0000 & 0.5000 & 2.4000 & 2.3400 & $2.50 \%$ & 2.4021 & $0.09 \%$ \\
\hline 24 & 5.7000 & 0.5000 & 2.3000 & 2.2300 & $3.04 \%$ & 2.3003 & $0.01 \%$ \\
\hline 25 & 6.6000 & 0.5000 & 2.2000 & 2.1000 & $4.55 \%$ & 2.2002 & $0.01 \%$ \\
\hline 26 & 7.5000 & 0.5000 & 2.1000 & 2.0000 & $4.76 \%$ & 2.1003 & $0.01 \%$ \\
\hline 27 & 7.7000 & 0.5000 & 2.1000 & 2.0000 & $4.76 \%$ & 2.0996 & $0.02 \%$ \\
\hline 28 & 1.0000 & 0.6000 & 2.9500 & 2.8900 & $2.03 \%$ & 2.9503 & $0.01 \%$ \\
\hline 29 & 2.0000 & 0.6000 & 2.8000 & 2.7700 & $1.07 \%$ & 2.7862 & $0.49 \%$ \\
\hline 30 & 3.0000 & 0.6000 & 2.6500 & 2.5700 & $3.02 \%$ & 2.6255 & $0.92 \%$ \\
\hline 31 & 4.0000 & 0.6000 & 2.5600 & 2.4580 & $3.98 \%$ & 2.4795 & $3.15 \%$ \\
\hline 32 & 5.0000 & 0.6000 & 2.4800 & 2.4400 & $1.61 \%$ & 2.3994 & $3.25 \%$ \\
\hline 33 & 5.7000 & 0.6000 & 2.3400 & 2.3000 & $1.71 \%$ & 2.3825 & $1.81 \%$ \\
\hline 34 & 6.6000 & 0.6000 & 2.2500 & 2.2000 & $2.22 \%$ & 2.2525 & $0.11 \%$ \\
\hline 35 & 7.5000 & 0.6000 & 2.1200 & 2.0000 & $5.66 \%$ & 2.1200 & $0.00 \%$ \\
\hline 36 & 7.7000 & 0.6000 & 2.1100 & 2.0000 & $5.21 \%$ & 2.1102 & $0.01 \%$ \\
\hline
\end{tabular}

Table 2 lists the generalization performance by ANN and SVR. From this table, it can be seen that the MAE, MAPE index estimated by SVRM is 0.0181 and $0.73 \%$, respectively, are both less than those of ANN model (0.0597, 2.46\%). This shows that the prediction errors based on SVRM model are smaller than those achieved by ANN model, the prediction accuracy of SVRM is greater than that of ANN. Table 2 also gives the multiple correlation coefficients of ANN and SVRM of 36 samples, and their $R^{2}$ are 0.958 and 0.983 respectively. It also reveals that the regression effect by SVRM is better than that of ANN model. 
Table 2: Comparison of prediction performance of ANN and SVRM

\begin{tabular}{cccc}
\hline Model & MAE (V) & MAPE & $R^{2}$ \\
\hline ANN & 0.0597 & $2.46 \%$ & 0.958 \\
SVRM & 0.0181 & $0.73 \%$ & 0.983 \\
\hline
\end{tabular}

Fig.1, shows the comparison between practical voltage and prediction voltage predicted by SVRM.

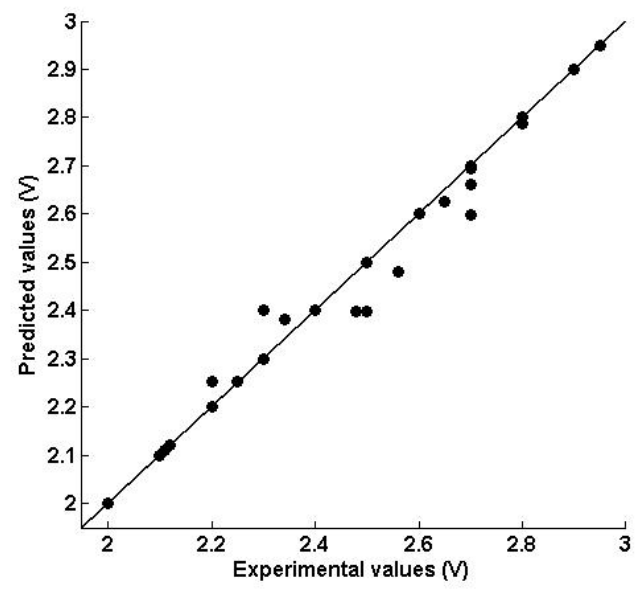

Fig. 1: Comparison of target values vs. estimated values predicted by SVRM

It is visible from Fig1 that nearly all the prediction points of SVRM model lie on or very close to the straight-line with slope 1 . This reflects that the PEMFC voltage values predicted by SVRM are in quite good agreement with the measured values.

\section{Conclusions}

Since the relation between stack voltage of proton exchange membrane fuel cell and its current density is nonlinear and influenced by hydrogen pressure, we use the SVRM, based on particle swarm optimization algorithm for its parameter optimization to develop a new prediction model for PEMFC. In the SVRM prediction model, PEMFC current density and hydrogen pressure were employed as input variables, the stack voltage act as output variables. The prediction result was provided by leave-one-out cross validation test of SVRM and compared with those of ANN model. The result shows that the accuracy of SVRM is higher enough to meet the demand of engineering and its prediction performance is better than ANN. These indicate that the black-box SVRM model can applies to the simulation and prediction of PEMFC.

\section{Acknowledgements}

The research work was supported by the Science and Technology Research Projects for the Guangxi Universities (YB 2014471).

\section{References}

[1] Mierlo, J. V. Van den Bossche, P. \& Maggetto, G., Models of energy sources for EV and HEV: fuel cells, batteries, ultra-capacitors, flywheels and engine-generators[J]. Journal Power Sources, 2004, 128 (1) 76-89.

[2] Martins, L.S. Gardolinski, J.E.F.C. Vargas, J.V.C. Ordonez, J.C. Amico, S.C. \& Forte, M.M.C., The experimental validation of a simplified PEMFC simulation model for design and optimization purposes[J] Applied Thermal Engineering, 2009, 29, 3036-3048.

[3] Manso,A.P. Marzo,F.F. Garmendia Mujika,M. Barranco,J. \& Lorenzo,A., Numerical analysis of the influence of the channel cross-section aspect ratio on the performance of a PEM fuel cell with serpentine flow field design[J], International Journal of Hydrogen Energy, 2011, 
36, 6795-6808.

[4] Sisworahardjo,N.S Yalcinoz,T. Sharkh,M.Y. \& Alam,M.S., Neural network model of 100W portable PEM fuel cell and experimental verification[J], International Journal of Hydrogen Energy, 2010, 35, 9104-9109 .

[3] Khajeh-Hosseini-Dalasm,N. Ahadian,S. Fushinobu, K. Okazaki,K. \& Kawazoe,Y., Prediction and analysis of the cathode catalyst layer performance of proton exchange membrane fuel cells using artificial neural network and statistical methods[J], Journal of Power Sources, 2011,196, 3750-3756.

[6] Vapnik,V., The nature of statistical learning theory, Springer, New York, 1995.

[7] Vapnik,V., An overview of statistical learning theory[J], IEEE Transaction on Neural Networks, 1999, 10, 988-999.

[8] Wen,Y.F. Cai,C.Z. Liu,X.H. Pei,J.F. Zhu,X.J. \&Xiao,T.T., Corrosion rate prediction of 3C steel under different seawater environment by using support vector regression[J], Corrosion Science, 2009, 51, 349-355.

[9] Khatibisepehr,S. Huang,B. Ibrahim,F. Xing,J.Z. \& Roa,W., Data-based modeling and prediction of cytotoxicity induced by contaminants in water resources[J], Computational Biology and Chemistry, 2011,35, 69-80.

[10] Nizam,M. Mohamed,A. \& Hussain,A., Dynamic voltage collapse prediction in power systems using support vector regression[J], Expert Systems with Applications, 2010, 37, 730-3736.

[11]Bhoopal,N. Venu Madhav,G. Pathapati,P.R. \& Dr. Amarnath,J., Modeling of Polymer Electrolyte Membrane Fuel Cell using Artificial Neural Networks[J]. International Journal of Recent Trends in Engineering, 2009, 2,75-79.

[12]Costamagna,P. Magistri,L. \& Massardo,A.F, Design and part-load performance of a hybrid system based on a solid oxide fuel cell reactor and a micro gas turbine[J], Journal Power Sources, 2001, 96, 352-368.

[13]Zhong,Z.D. Zhua,X.J. \& Cao,G.Y., Modeling a PEMFC by a support vector machine[J], Journal of Power Sources, 2006, 160, 293-298. 